\title{
THE TILTED CARATHÉODORY CLASS AND ITS APPLICATIONS
}

\author{
Li-MEI WANG
}

\begin{abstract}
This paper mainly deals with the tilted Carathéodory class by angle $\lambda \in(-\pi / 2, \pi / 2)$ (denoted by $\mathcal{P}_{\lambda}$ ) an element of which maps the unit disc into the tilted right half-plane $\left\{w: \operatorname{Re} e^{i \lambda} w>0\right\}$. Firstly we will characterize $\mathcal{P}_{\lambda}$ from different aspects, for example by subordination and convolution. Then various estimates of functionals over $\mathcal{P}_{\lambda}$ are deduced by considering these over the extreme points of $\mathcal{P}_{\lambda}$ or the knowledge of functional analysis. Finally some subsets of analytic functions related to $\mathcal{P}_{\lambda}$ including close-to-convex functions with argument $\lambda$, $\lambda$-spirallike functions and analytic functions whose derivative is in $\mathcal{P}_{\lambda}$ are also considered as applications.
\end{abstract}

\section{Introduction}

Let $\mathcal{A}$ be the family of functions $f$ analytic in the unit disc $\mathbb{D}=\{z \in$ $\mathbb{C}:|z|<1\}$, and $\mathcal{A}_{1}$ be the subset of $\mathcal{A}$ consisting of functions $f$ which are normalized by $f(0)=f^{\prime}(0)-1=0$ while $\mathcal{A}_{0}$ with normalization $f(0)=1$. A function $f \in \mathcal{A}$ is said to be subordinate to a function $F \in \mathcal{A}$ (in symbols $f \prec F$ or $f(z) \prec F(z))$ in $\mathbb{D}$ if there exists an analytic function $\omega$ on $\mathbb{D}$ with $|\omega(z)|<1$ and $\omega(0)=0$, such that

$$
f(z)=F(\omega(z))
$$

in $\mathbb{D}$. When $F$ is a univalent function, the condition $f \prec F$ is equivalent to $f(\mathbb{D}) \subseteq F(\mathbb{D})$ and $f(0)=F(0)$.

Let

$$
\mathcal{P}_{\lambda}=\left\{p \in \mathcal{A}_{0}: \operatorname{Re} e^{i \lambda} p(z)>0\right\} .
$$

Here and hereafter we always suppose $-\pi / 2<\lambda<\pi / 2$. Note that $\mathcal{P}_{\lambda}$ is a convex and compact subset of $\mathcal{A}$ which is equipped with the topology of uniform convergence on compact subsets of $\mathbb{D}$. Since $\mathcal{P}_{0}$ is the well-known Carathéodory

Received July 22, 2010; Revised August 26, 2010.

2010 Mathematics Subject Classification. Primary 30C45; Secondary 30C70.

Key words and phrases. the tilted Carathéodory class, $\lambda$-spirallike functions, close-toconvex functions with argument $\lambda$, convolution, subordination. 
class, we call $\mathcal{P}_{\lambda}$ the tilted Carathéodory class by angle $\lambda$. Also we let

$$
\mathcal{P}=\bigcup_{-\pi / 2<\lambda<\pi / 2} \mathcal{P}_{\lambda}
$$

In this note, we always let

$$
p_{\lambda}(z)=\frac{1+e^{-2 i \lambda} z}{1-z}
$$

It is easy to see that $p_{\lambda}$ univalently maps the unit disc $\mathbb{D}$ onto $\mathbb{H}_{\lambda}=\{w \in$ $\left.\mathbb{C}: \operatorname{Re} e^{i \lambda} w>0\right\}$ which is called the tilted right half-plane by angle $\lambda$. Later we will see that this function plays an important role while investigating the properties of $\mathcal{P}_{\lambda}$.

The Carathéodory class $\mathcal{P}_{0}$ occupies an extremely important place in the theory of functions and has been studied by many authors ([5, Chapter 7, p. 77], [6], [16], [19], [20], [23], [24], [29], [30]). The tilted Carathéodory class $\mathcal{P}_{\lambda}$ scatters in some papers (see [13], [14], [16], [21]), although the name was not given in the literature. In the geometric function theory, there are some functions defined by using the tilted Carathéodory class $\mathcal{P}_{\lambda}$, such as the class of close-to-convex functions. When researching this class, some authors restrict to the special case $\lambda=0$ because of a difficulty lying in $\mathcal{P}_{\lambda}$. Therefore it is worthwhile investigating the class $\mathcal{P}_{\lambda}$ in order to understand its relating geometric functions well.

Section 2 is devoted to characterizations of the functions belonging to the class $\mathcal{P}_{\lambda}$ from different aspects. A linear relation between the elements of $\mathcal{P}_{\lambda}$ and $\mathcal{P}_{0}$ implies that the functions in $\mathcal{P}_{\lambda}$ can be described in terms of integral and subordination. We show also that $\mathcal{P}_{\lambda}$ can be regarded as the dual and the second dual sets of some families of analytic functions.

In Section 3, the extreme points of $\mathcal{P}_{\lambda}$ are deduced directly from those of $\mathcal{P}_{0}$. With the aid of these extreme points, the sharp estimates of some functionals over $\mathcal{P}_{\lambda}$, for instance, the $n$-th coefficient functional, the distortion and growth functionals, have been obtained. For the cases of other functionals, we summarize some other methods to deal with the extremal problems. The estimate of $\left|z p^{\prime}(z) /(p(z)+i \tan \lambda)\right|$ for $p \in \mathcal{P}_{0}$ was considered by some authors, see Bernardi [2] and Robertson [20]. The sharp estimate was obtained in a paper of Ruscheweyh and Singh [23] by a variational method but the extremal functions were not given there. In Theorem 6 of the present paper, we estimate the functional $\left|z p^{\prime}(z) / p(z)\right|$ with $p \in \mathcal{P}_{\lambda}$ which is actually equivalent to the above problem. By using fundamental functional analysis, we obtain the sharp upper bound of $\left|z p^{\prime}(z) / p(z)\right|$ for $p \in \mathcal{P}_{\lambda}$ and give all the extremal functions which make the estimate sharp.

The last section is concerned with some applications of our results to Geometric Function Theory. We will consider $\lambda$-spirallike functions, close-to-convex functions with argument $\lambda$ and analytic functions whose derivative is in $\mathcal{P}_{\lambda}$. 


\section{Characterizations of $\mathcal{P}_{\lambda}$}

In this section, we list some characterizations of $\mathcal{P}_{\lambda}$ for later use. Before proceeding to it, we shall first introduce some notations. The convolution (or Hadamard product) $f * g$ of two functions $f, g \in \mathcal{A}$ with series expansions $f(z)=\sum_{n=0}^{\infty} a_{n} z^{n}$ and $g(z)=\sum_{n=0}^{\infty} b_{n} z^{n}$ is defined by

$$
f * g(z)=\sum_{n=0}^{\infty} a_{n} b_{n} z^{n} .
$$

Obviously, we have $f * g \in \mathcal{A}$. For an introduction to the theory of convolutions in the present context we refer to [22]. For an analytic function $h \in \mathcal{A}_{0}$, we note that

$$
\begin{aligned}
h(z) * \frac{1+A z}{1-z} & =h(z) *\left(\frac{1+A}{1-z}-A\right) \\
& =(1+A) h(z)-A
\end{aligned}
$$

for a complex number $A$ which will be used several times. For a set $V \subset \mathcal{A}_{0}$, define the dual set

$$
V^{*}=\left\{g \in \mathcal{A}_{0}:(f * g)(z) \neq 0 \text { in } \mathbb{D} \text { for any } f \in V\right\},
$$

and $V^{* *}=\left(V^{*}\right)^{*}$, the second dual.

The next lemma can be deduced from the proof of Theorem 1.3 in [22].

Lemma 1. Let $h \in \mathcal{A}_{0}$. If

$$
t h(x z)+(1-t) h(y z) \neq 0
$$

for any $|x|=|y|=1,0 \leq t \leq 1$ and $z \in \mathbb{D}$, then $h(\mathbb{D})$ is contained in a half-plane $H$ with $0 \in \partial H$.

Theorem 1. Let $\lambda \in(-\pi / 2, \pi / 2)$ be a real constant. Then the following conditions are equivalent for a function $p \in \mathcal{A}_{0}$

(i) $p \in \mathcal{P}_{\lambda}$;

(ii) $\frac{e^{i \lambda} p-i \sin \lambda}{\cos \lambda} \in \mathcal{P}_{0}$;

(iii) There exists a Borel probability measure $\mu$ on $\partial \mathbb{D}$ such that $p(z)$ can be represented by

$$
p(z)=\int_{\partial \mathbb{D}} \frac{1+e^{-2 i \lambda} x z}{1-x z} d \mu(x)
$$

(iv) $p \prec p_{\lambda}$ in $\mathbb{D}$;

(v) $p \in V_{\lambda}^{*}$, where

$$
V_{\lambda}=\left\{\frac{1}{1-z}\left(1+\frac{1-e^{-2 i \lambda} x}{\left(1+e^{-2 i \lambda}\right) x} z\right):|x|=1\right\}
$$


(vi) $p \in W_{\lambda}^{* *}$, where

$W_{\lambda}=\left\{t \frac{1+x e^{-2 i \lambda} z}{1-x z}+(1-t) \frac{1+y e^{-2 i \lambda} z}{1-y z}:|x|=|y|=1,0 \leq t \leq 1\right\}$.

Remark 1. Theorem 1 implies that

$$
\mathcal{P}_{\lambda}=V_{\lambda}^{*}
$$

and

$$
\mathcal{P}_{\lambda}=W_{\lambda}^{* *} .
$$

For $\mathcal{P}$, we have (see [22, Theorem 1.6])

$$
\mathcal{P}=\left\{f \in \mathcal{A}_{0}: \operatorname{Re} f(z)>1 / 2\right\}^{*}
$$

and

$$
\mathcal{P}=\left\{\frac{1+x z}{1+y z}:|x|=|y|=1\right\}^{* *} .
$$

Proof of Theorem 1. The equivalence of (i), (ii) and (iv) can be obtained immediately from the definition of $\mathcal{P}_{\lambda}$. The condition (iii) is reduced to the Herglotz integral representation of $\mathcal{P}_{0}([9]$, see also [8]) when $\lambda=0$. Thus by the equivalence of (i) and (ii), we can easily get (i) $\Leftrightarrow$ (iii). Hence we only need to prove the equivalence of (i), (v) and (vi).

Firstly we will show $(\mathrm{i}) \Leftrightarrow(\mathrm{v})$. We have to prove that

$$
p(z) * \frac{1}{1-z}\left(1+\frac{1-e^{-2 i \lambda} x}{\left(1+e^{-2 i \lambda}\right) x} z\right) \neq 0,|x|=1, z \in \mathbb{D}
$$

if and only if $p \in \mathcal{P}_{\lambda}$.

By making use of relation (1), we see that (2) is equivalent to

$$
p(z) *\left(\frac{1}{1-z}-\frac{1-e^{-2 i \lambda} x}{1+x}\right) \neq 0,
$$

namely,

$$
p(z) \neq \frac{1-e^{-2 i \lambda} x}{1+x}
$$

for $|x|=1, x \neq-1$ and $z \in \mathbb{D}$. Since the set

$$
\left\{\left(1-e^{-2 i \lambda} x\right) /(1+x):|x|=1, x \neq-1\right\}
$$

is just the line $\left\{w: \operatorname{Re} e^{i \lambda} w=0\right\}$, the above condition implies that $p(\mathbb{D})$ lies in the tilted right half-plane by angle $\lambda$, which is our assertion.

Next we will show (i) $\Leftrightarrow($ vi). To this end, we need to prove that an analytic function $h \in \mathcal{A}_{0}$ satisfies

$$
h(z) *\left(t \frac{1+x e^{-2 i \lambda} z}{1-x z}+(1-t) \frac{1+y e^{-2 i \lambda} z}{1-y z}\right) \neq 0
$$

for any $|x|=|y|=1,0 \leq t \leq 1$ and $z \in \mathbb{D}$, if and only if

$$
h * p(z) \neq 0
$$


for $z \in \mathbb{D}$ and $p \in \mathcal{P}_{\lambda}$.

If an analytic function $h$ with $h(0)=1$ satisfies (3) for any $|x|=|y|=1$, $0 \leq t \leq 1$ and $z \in \mathbb{D}$, then by relation (1), we have

$$
t h(x z)+(1-t) h(y z) \neq \frac{e^{-2 i \lambda}}{1+e^{-2 i \lambda}}
$$

for any $|x|=|y|=1,0 \leq t \leq 1$ and $z \in \mathbb{D}$. Thus by Lemma 1 , there exists a real constant $\alpha \in[0,2 \pi)$ such that

$$
\operatorname{Re}\left(e^{i \alpha}\left(h(z)-\frac{e^{-2 i \lambda}}{1+e^{-2 i \lambda}}\right)\right)>0
$$

which implies that for any Borel probability measure $\mu$ on $\partial \mathbb{D}$ we have

$$
\int_{\partial \mathbb{D}}\left(h(x z)-\frac{e^{-2 i \lambda}}{1+e^{-2 i \lambda}}\right) d \mu(x) \neq 0,
$$

equivalently,

$$
h(z) * \int_{\partial \mathbb{D}} \frac{1+e^{-2 i \lambda} x z}{1-x z} d \mu(x) \neq 0 .
$$

Then for any function $p \in \mathcal{P}_{\lambda}$ we have

$$
p * h(z) \neq 0
$$

for $z \in \mathbb{D}$ by the equivalence of (i) and (iii). Since the above process is invertible, we thus arrive at our conclusions.

By making use of the following Schur's lemma, we can get a convolution property of $\mathcal{P}_{\lambda}$.

Lemma 2 (see [25] or [15]). Let $p_{1}(z)=1+\sum_{n=1}^{\infty} a_{n} z^{n} \in \mathcal{P}_{0}$ and $p_{2}(z)=$ $1+\sum_{n=1}^{\infty} b_{n} z^{n} \in \mathcal{P}_{0}$. Then

$$
1+\sum_{n=1}^{\infty} a_{n} b_{n} z^{n} / 2 \in \mathcal{P}_{0}
$$

Theorem 2. Let $p_{1} \in \mathcal{P}_{\lambda_{1}}$ and $p_{2} \in \mathcal{P}_{\lambda_{2}}$. Then

$$
\operatorname{Re}\left(e^{i\left(\lambda_{1}+\lambda_{2}\right)}\left(p_{1} * p_{2}\right)\right)>-\cos \left(\lambda_{1}-\lambda_{2}\right) .
$$

In particular, if $\cos \left(\lambda_{1}-\lambda_{2}\right)<0$, then

$$
p_{1} * p_{2} \in \mathcal{P}_{\lambda_{1}+\lambda_{2}} .
$$

Proof. Since $p_{1}(z) \in \mathcal{P}_{\lambda_{1}}$ and $p_{2}(z) \in \mathcal{P}_{\lambda_{2}}$, by the equivalence (i) and (ii) in Theorem 1 we have

and

$$
\frac{e^{i \lambda_{1}} p_{1}(z)-i \sin \lambda_{1}}{\cos \lambda_{1}} \in \mathcal{P}_{0}
$$

$$
\frac{e^{i \lambda_{2}} p_{2}(z)-i \sin \lambda_{2}}{\cos \lambda_{2}} \in \mathcal{P}_{0} .
$$


Hence Schur's lemma implies that

$$
\frac{1}{2}\left(\frac{e^{i \lambda_{1}} p_{1}(z)-i \sin \lambda_{1}}{\cos \lambda_{1}} * \frac{e^{i \lambda_{2}} p_{2}(z)-i \sin \lambda_{2}}{\cos \lambda_{2}}\right)+\frac{1}{2} \in \mathcal{P}_{0}
$$

which is equivalent to (4).

\section{Basic estimates of $\mathcal{P}_{\lambda}$}

Let $K$ be a subset of a vector space $X$. A point $s$ in $K$ is called an extreme point if it is not an internal point of any line interval whose endpoints are in $K$, except when both endpoints are $s$. We denote the set of all extreme points of $K$ by Ext $K$. The extremal points of $\mathcal{P}_{0}$ can be obtained by the Herglotz integral representation formula. A truly beautiful derivation of $\operatorname{Ext} \mathcal{P}_{0}$ was given by Holland [10], while Kortram [15] obtained it by elementary functional analysis. We state it as a lemma in order to get the corresponding result of $\mathcal{P}_{\lambda}$.

Lemma 3.

$$
\operatorname{Ext} \mathcal{P}_{0}=\left\{\frac{1+x z}{1-x z},|x|=1\right\}
$$

Theorem 3.

$$
\operatorname{Ext} \mathcal{P}_{\lambda}=\left\{p_{\lambda}(x z),|x|=1\right\}
$$

Proof. A combination of Lemma 3 and the equivalence of (i) and (ii) in Theorem 1 implies our assertion.

The next result which can be found in [8, p. 45] gives a useful technique to solve extremal problems. If $\mathcal{F}$ is a convex subset of $\mathcal{A}$ and $J: \mathcal{A} \rightarrow \mathbb{R}$, then $J$ is called convex on $\mathcal{F}$ provided that $J(t f+(1-t) g) \leq t J(f)+(1-t) J(g)$ whenever $f, g \in \mathcal{F}$ and $0 \leq t \leq 1$.

Lemma 4. Let $\mathcal{F}$ be a compact and convex subset of $\mathcal{A}$ and let $J$ be a realvalued, continuous, convex functional on $\mathcal{F}$. Then

$$
\max \{J(f): f \in \mathcal{F}\}=\max \{J(f): f \in \operatorname{Ext} \mathcal{F}\} .
$$

Theorem 4. Let $p(z)=1+\sum_{n=1}^{\infty} p_{n} z^{n} \in \mathcal{P}_{\lambda}$ with $\lambda \in(-\pi / 2, \pi / 2)$. Then

$$
\left|p_{n}\right| \leq 2 \cos \lambda \text {, }
$$

and

$$
\left|p^{\prime}(z)\right| \leq \frac{2 \cos \lambda}{(1-r)^{2}}
$$

where $r=|z|<1$. The inequalities are sharp with the extremal functions $p_{\lambda}(x z)$, where $|x|=1$.

Proof. It is easy to check that the above two functionals are real-valued, continuous and convex. Thus by applying Lemma 4 the maxima of them are 
obtained over the reduced subset Ext $\mathcal{P}_{\lambda}$, which consists of $p_{\lambda}(x z)$ with $|x|=1$ by Theorem 3 . Since

$$
p_{\lambda}(x z)=1+\left(1+e^{2 i \lambda}\right) \sum_{n=1}^{\infty} x^{n} z^{n}
$$

and

$$
p_{\lambda}^{\prime}(x z)=\frac{1+e^{2 i \lambda}}{(1-x z)^{2}}
$$

we complete the proof.

Theorem 5. Let $p \in \mathcal{P}_{\lambda}$ with $\lambda \in(-\pi / 2, \pi / 2)$. Then

$$
\left|p(z)-\frac{1+r^{2} e^{-2 i \lambda}}{1-r^{2}}\right| \leq \frac{2 r \cos \lambda}{1-r^{2}},
$$

where $r=|z|<1$. In particular, we have

$$
\frac{1+r^{2} \cos 2 \lambda-2 r \cos \lambda}{1-r^{2}} \leq \operatorname{Re} p(z) \leq \frac{1+r^{2} \cos 2 \lambda+2 r \cos \lambda}{1-r^{2}}
$$

and

$$
1 / A(\lambda, r) \leq|p(z)| \leq A(\lambda, r),
$$

where $A(\lambda, r)$ is given by

$$
A(\lambda, r)=\frac{\sqrt{\left(1-r^{2}\right)^{2}+4 r^{2} \cos ^{2} \lambda}+2 r \cos \lambda}{1-r^{2}} .
$$

These inequalities are sharp with the extremal functions $p_{\lambda}(x z)$, where $|x|=1$.

Proof. By using the same arguments as in Theorem 4, the maximum of the first functional over $\mathcal{P}_{\lambda}$ is obtained over the set of $p_{\lambda}(x z)$ with $|x|=1$. Therefore

$$
\begin{aligned}
\left|p_{\lambda}(x z)-\frac{1+r^{2} e^{-2 i \lambda}}{1-r^{2}}\right| & =\left|\frac{\left(1+e^{2 i \lambda}\right)\left(x z-r^{2}\right)}{(1-x z)\left(1-r^{2}\right)}\right| \\
& =\frac{2 \cos \lambda}{1-r^{2}}\left|\frac{x z-r^{2}}{1-x z}\right| \\
& =\frac{2 r \cos \lambda}{1-r^{2}},
\end{aligned}
$$

where $r=|z|<1$ and $|x|=1$, which is what we want. The other estimates can be deduced directly from the first one.

Remark 2. The first inequality in Theorem 5 implies that the function $p \in \mathcal{P}_{\lambda}$ maps the disc $|z|<r$ into $U(\lambda, r)$ the hyperbolic disc in the tilted half plane $\mathbb{H}_{\lambda}$ centered at 1 with radius $\operatorname{arctanh} r$.

Although the extremal problems of real-valued continuous convex functionals over $\mathcal{P}_{\lambda}$ can be solved within the set $p_{\lambda}(x z),|x|=1$, it is not applicable 
for general functionals. Robertson [19], [20] and Sakaguchi [24] obtained variational formulae for $\mathcal{P}_{0}$ and showed that, for fixed $z \in \mathbb{D}$, the extremal values of

$$
\operatorname{Re} F\left(p(z), z p^{\prime}(z)\right), p \in \mathcal{P}_{0},
$$

where $F(u, v)$ is analytic in $(u, v) \in \mathbb{C}^{2}$, $\operatorname{Re} u>0$, are always attained by the functions

$$
t\left(\frac{1+x z}{1-x z}\right)+(1-t)\left(\frac{1+\bar{x} z}{1-\bar{x} z}\right), 0 \leq t \leq 1,|x|=1 .
$$

Since there exists a linear relation between $\mathcal{P}_{0}$ and $\mathcal{P}_{\lambda}$, we can see that for a fixed $z \in \mathbb{D}$, the extremal values of

$$
\operatorname{Re} F\left(p(z), z p^{\prime}(z)\right), p \in \mathcal{P}_{\lambda},
$$

where $F(u, v)$ is analytic in $(u, v) \in \mathbb{C}^{2}$, $\operatorname{Re} e^{i \lambda} u>0$, are attained by the functions

$$
t\left(\frac{1+x e^{-2 i \lambda} z}{1-x z}\right)+(1-t)\left(\frac{1+\bar{x} e^{-2 i \lambda} z}{1-\bar{x} z}\right), 0 \leq t \leq 1,|x|=1 .
$$

For another functional

$$
\frac{F_{1}(p)}{F_{2}(p)},
$$

where $F_{1}$ and $F_{2}$ are real-valued continuous linear functionals over $\mathcal{P}_{\lambda}$ with $F_{2}(p) \neq 0$ for $p \in \mathcal{P}_{\lambda}$, it follows from the Duality Principle [18, Corollary 1.1] and Remark 1 that the extremal value of it is attained by a function in $W_{\lambda}$. However, for many cases of interest, it is not easy to obtain extremal values even for those restricted classes of functions, for instance the functional $z p^{\prime}(z) / p(z)$ over $\mathcal{P}_{\lambda}$. Our next theorem solves this problem by using elementary functional analysis.

Theorem 6. Let $p \in \mathcal{P}_{\lambda}$ with $\lambda \in(-\pi / 2, \pi / 2)$. Then

$$
\left|\frac{z p^{\prime}(z)}{p(z)}\right| \leq M(\lambda,|z|)
$$

where

$$
M(\lambda, r)=\left\{\begin{array}{l}
\frac{2 r \cos \lambda}{1+r^{2}-2 r|\sin \lambda|}, \quad r<|\tan (\lambda / 2)|, \\
\frac{2 r}{1-r^{2}}, \quad r \geq|\tan (\lambda / 2)| .
\end{array}\right.
$$

Equality holds for some point $z_{0}=r e^{i \theta}, 0<r<1$, if and only if $p(z)=p_{\lambda}(x z)$ where $x=e^{i(\alpha-\theta)}$ with $\alpha$ satisfying

$$
\left\{\begin{array}{l}
\alpha=\pi / 2+\lambda, \quad r<-\tan (\lambda / 2) \\
\alpha=-\pi / 2+\lambda, \quad r<\tan (\lambda / 2) \\
\sin (\alpha-\lambda)=-\frac{1+r^{2}}{1-r^{2}} \sin \lambda, \quad r \geq|\tan (\lambda / 2)| .
\end{array}\right.
$$


Remark 3. For a fixed $0<r<1, M(\lambda, r)$ is a symmetric function in $\lambda$ with respect to the origin and it is also decreasing in $0 \leq \lambda<\pi / 2$. We thus have $M(\lambda, r) \leq M(0, r)=2 r /\left(1-r^{2}\right)$ for any $\lambda \in(-\pi / 2, \pi / 2)$ which is a known result for Gelfer functions ([5, p. 73], see also [28], [4] or [12]).

In order to prove the above theorem, the following lemma is needed.

\section{Lemma 5.}

$$
N(\lambda, r) \leq\left|\frac{z p_{\lambda}^{\prime}(z)}{p_{\lambda}(z)}\right| \leq M(\lambda, r)
$$

for $r=|z|<1$, where $M(\lambda, r)$ is defined by (6) and

$$
N(\lambda, r)=\frac{2 r \cos \lambda}{1+r^{2}+2 r|\sin \lambda|} .
$$

The equality in the right-hand side holds at $z_{0}=r e^{i \theta}$ with $\theta$ in place of $\alpha$ satisfying (7) while the other side holds at $z_{0}=r e^{i \theta}$ with $\theta$ satisfying

$$
\left\{\begin{array}{l}
\theta-\lambda=\pi / 2, \quad \lambda>0, \\
\theta-\lambda=-\pi / 2, \quad \lambda \leq 0 .
\end{array}\right.
$$

Proof. By observing that

$$
\left|\frac{\bar{z} p_{\lambda}^{\prime}(\bar{z})}{p_{\lambda}(\bar{z})}\right|=\left|\frac{z p_{-\lambda}^{\prime}(z)}{p_{-\lambda}(z)}\right|,
$$

we can restrict ourselves to the case $\lambda \geq 0$.

Since $p_{\lambda}^{\prime}(z) / p_{\lambda}(z)=\left(1+e^{2 i \lambda}\right) /(1-z)\left(e^{2 i \lambda}+z\right)$, after letting $z=r e^{i(\alpha+\lambda+\pi / 2)}$ and $h(\alpha)=\left|(1-z)\left(e^{2 i \lambda}+z\right)\right|^{2}=\left|\left(1-r e^{i(\alpha+\lambda+\pi / 2)}\right)\left(e^{2 i \lambda}+r e^{i(\alpha+\lambda+\pi / 2)}\right)\right|^{2}$, we obtain

$$
h(\alpha)=\left(1+r^{2}+2 r \sin (\alpha+\lambda)\right)\left(1+r^{2}+2 r \sin (\lambda-\alpha)\right)
$$

and

$$
\frac{2 r \cos \lambda}{\max _{-\pi<\alpha \leq \pi} \sqrt{h(\alpha)}} \leq\left|\frac{z p_{\lambda}^{\prime}(z)}{p_{\lambda}(z)}\right| \leq \frac{2 r \cos \lambda}{\min _{-\pi<\alpha \leq \pi} \sqrt{h(\alpha)}} .
$$

It is thus sufficient to search for the maximum and minimum of $h(\alpha)$ over $-\pi<\alpha \leq \pi$. A simple calculation yields

$$
h^{\prime}(\alpha)=-4 r \sin \alpha\left[\left(1+r^{2}\right) \sin \lambda+2 r \cos \alpha\right] .
$$

Since $h(\alpha)$ is smooth and periodic, the candidate minimum points of $h(\alpha)$ are the zero points of $h^{\prime}(\alpha)$ which are $\alpha_{1}=0, \alpha_{2}=\pi$ and $\alpha_{3}= \pm \arccos (-(1+$ $\left.\left.r^{2}\right) \sin \lambda / 2 r\right)$. Here $\alpha_{3}$ is meaningful only when $\sin \lambda \leq 2 r /\left(1+r^{2}\right)$, namely $r \geq \tan (\lambda / 2)$. A calculation gives

and

$$
\begin{aligned}
& h(0)=\left(1+r^{2}+2 r \sin \lambda\right)^{2}, \\
& h(\pi)=\left(1+r^{2}-2 r \sin \lambda\right)^{2},
\end{aligned}
$$

$$
h\left(\alpha_{3}\right)=\cos ^{2} \lambda\left(1-r^{2}\right)^{2} .
$$


$\lambda \geq 0$ implies that $h(\pi) \leq h(0)$. Since $h(\pi) \geq h\left(\alpha_{3}\right)$, we can get the minimum of $h(\alpha)$ is

$$
\left\{\begin{array}{l}
\left(1+r^{2}-2 r \sin \lambda\right)^{2}, \quad r<\tan (\lambda / 2) \\
\cos ^{2} \lambda\left(1-r^{2}\right)^{2}, \quad r \geq \tan (\lambda / 2)
\end{array}\right.
$$

and the maximum of $h(\alpha)$ is

$$
\left(1+r^{2}+2 r \sin \lambda\right)^{2} .
$$

Finally by using (9), (10) and (11), we can obtain our claims immediately.

Proof of Theorem 6. The equivalence of (i) and (iv) in Theorem 1 implies that if $p \in \mathcal{P}_{\lambda}$, then there exists a function $\omega \in \mathcal{A}$ with $|\omega(z)|<1$ and $\omega(0)=0$ such that

$$
p(z)=p_{\lambda}(\omega(z))
$$

in $\mathbb{D}$. Then by making use of Lemma 5 and the Schwarz-Pick lemma, we have

$$
\begin{aligned}
\left|\frac{z p^{\prime}(z)}{p(z)}\right| & =\left|\frac{z \omega^{\prime}(z) p_{\lambda}^{\prime}(\omega(z))}{p_{\lambda}(\omega(z))}\right|=\left|\frac{z \omega^{\prime}}{\omega}\right|\left|\frac{\omega^{\prime} p_{\lambda}^{\prime}(\omega)}{p_{\lambda}(\omega)}\right| \\
& \leq\left\{\begin{array}{l}
\frac{1-|\omega|^{2}}{1-|z|^{2}} \frac{2|z| \cos \lambda}{1+|\omega|^{2}-2|\omega||\sin \lambda|}, \quad|\omega|<\tan (\lambda / 2), \\
\frac{1-|\omega|^{2}}{1-|z|^{2}} \frac{2|z|}{1-|\omega|^{2}}, \quad|\omega| \geq \tan (\lambda / 2) .
\end{array}\right.
\end{aligned}
$$

Since $|\omega(z)| \leq|z|=r$, the function $2|\omega| /\left(1+|\omega|^{2}\right)$ is increasing in $|\omega| \in[0, r]$ and attains its maximum value $2 r /\left(1+r^{2}\right)$ if and only if $\omega(z) \equiv x z$ with $|x|=1$. On the other hand,

$$
\frac{1-|\omega|^{2}}{1-r^{2}} \frac{2 r \cos \lambda}{1+|\omega|^{2}-2|\omega||\sin \lambda|}
$$

is also increasing in $|\omega|$ provided $|\omega|<|\tan (\lambda / 2)|$. Therefore inequality (12) implies

$$
\left|\frac{z p^{\prime}(z)}{p(z)}\right| \leq\left\{\begin{array}{l}
\frac{2 r \cos \lambda}{1+r^{2}-2 r|\sin \lambda|}, \quad r<|\tan (\lambda / 2)|, \\
\frac{2 r}{1-r^{2}}, \quad r \geq|\tan (\lambda / 2)| .
\end{array}\right.
$$

Hence the proof of Theorem 6 is completed.

The sharp estimate of the entity in Theorem 6 first appears in the following form in a paper [23] by Ruscheweyh and Singh. Their proof was based on a variational method.

Theorem A. For $p \in \mathcal{P}_{0}$ and $\lambda \in(-\pi / 2, \pi / 2)$ the estimate

$$
\left|\frac{z p^{\prime}(z)}{p(z)+i \tan \lambda}\right| \leq \begin{cases}\frac{\left(1-|z|^{2}\right) \cos \lambda}{1-2|z||\sin \lambda|+|z|^{2}}, & |z|<\left|\tan \frac{\lambda}{2}\right|, \\ 1, & |z| \geq\left|\tan \frac{\lambda}{2}\right|,\end{cases}
$$


is valid and sharp. Equality holds for certain functions in $\mathcal{P}_{0}$.

Note that Theorem 6 improves Theorem A since it shows that the extremal functions are only $p_{\lambda}(x z)$ with $|x|=1$.

Proposition 1. Let $p \in \mathcal{P}_{\lambda}$ with $\lambda \in(-\pi / 2, \pi / 2)$. Then

$$
\left|\operatorname{Im} \frac{z p^{\prime}(z)}{p(z)}\right| \leq M(\lambda, r)
$$

and

$$
\left|\operatorname{Re} \frac{z p^{\prime}(z)}{p(z)}\right| \leq M(\lambda, r)
$$

where $r=|z|<1$ and $M(\lambda, r)$ is given in (6). Equality occurs at point $z_{0}=r e^{i \theta}$ in the first inequality if and only if $p(z)=p_{\lambda}(x z)$ and $r \leq|\tan (\lambda / 2)|$, where $x=e^{i(\alpha-\theta)}$ with $\alpha$ satisfying $(7)$.

Proof. Since the above inequalities are straightforward consequences of Theorem 6 , we only need to verify the sharpness. By a simple calculation, it is easy to see that if $\lambda<0$ for any fixed $r \leq-\tan (\lambda / 2)$

$$
\frac{z p_{\lambda}^{\prime}(z)}{p_{\lambda}(z)}=\frac{z\left(1+e^{2 i \lambda}\right)}{(1-z)\left(e^{2 i \lambda}+z\right)}=\frac{-2 r i \cos \lambda}{1+r^{2}-2 r \sin \lambda}=-i M(\lambda, r)
$$

when $z=i r e^{i \lambda}$. Similarly, we can get if $\lambda \geq 0$ for any fixed $r \leq \tan (\lambda / 2)$

$$
\frac{z p_{\lambda}^{\prime}(z)}{p_{\lambda}(z)}=\frac{2 r i \cos \lambda}{1+r^{2}-2 r \sin \lambda}=i M(\lambda, r)
$$

when $z=-i r e^{i \lambda}$. Our proof is completed.

We shall conclude this section with a result due to Kim and Sugawa [14] which gives a sufficient condition for membership of $\mathcal{P}_{\lambda}$. Note that the function $z p_{\lambda}^{\prime}(z) / p_{\lambda}(z)$ maps $\mathbb{D}$ univalently onto $U_{\lambda}$, where $U_{\lambda}$ is the slit domain defined by

$$
U_{\lambda}=\mathbb{C} \backslash\left\{i y: y \geq A_{\lambda} \text { or } y \leq-1 / A_{\lambda}\right\}, A_{\lambda}=\cos \lambda /(1+\sin \lambda) .
$$

Since $U_{\lambda}$ is a starlike domain, the function $z p_{\lambda}^{\prime}(z) / p_{\lambda}(z)$ is starlike. Hence by using Lemma 3 of [14], we can obtain:

Theorem 7. Let $p \in \mathcal{A}_{0}$ satisfy the subordination

$$
\frac{z p^{\prime}(z)}{p(z)} \prec \frac{z p_{\lambda}^{\prime}(z)}{p_{\lambda}(z)}
$$

in $\mathbb{D}$. Then $p \in \mathcal{P}_{\lambda}$. 


\section{Applications}

\section{1. $\lambda$-spirallike functions}

Definition 1 ([3], see also [1]). A function $f \in \mathcal{A}_{1}$ is called $\lambda$-spirallike (denoted by $f \in \mathcal{S P}(\lambda))$ for a real number $\lambda \in(-\pi / 2, \pi / 2)$ if

$$
\frac{z f^{\prime}}{f} \in \mathcal{P}_{\lambda}
$$

Spirallike functions are shown to be univalent by Špaček [26]. Note that $\mathcal{S P}(0)$ is precisely the set of starlike functions normally denoted by $\mathcal{S}^{*}$.

By the definition of $\lambda$-spirallike function, we can easily deduce the following corollary from Theorem 5:

Corollary 1. Let $f \in \mathcal{S P}(\lambda)$. Then

$$
\left|\frac{z f^{\prime}(z)}{f(z)}-\frac{1+r^{2} e^{-2 i \lambda}}{1-r^{2}}\right| \leq \frac{2 r \cos \lambda}{1-r^{2}},
$$

where $r=|z|<1$. In particular, we have

$$
\frac{1+r^{2} \cos 2 \lambda-2 r \cos \lambda}{1-r^{2}} \leq \operatorname{Re} \frac{z f^{\prime}(z)}{f(z)} \leq \frac{1+r^{2} \cos 2 \lambda+2 r \cos \lambda}{1-r^{2}}
$$

and

$$
1 / A(\lambda, r) \leq\left|\frac{z f^{\prime}(z)}{f(z)}\right| \leq A(\lambda, r),
$$

where $A(\lambda, r)$ is given by (5). Those inequalities are sharp with the extremal function given by

$$
f_{\lambda}(z)=\frac{z}{(1-z)^{1+e^{-2 i \lambda}}} .
$$

Note that the lower bound of the second estimate was proved by Robertson [21], but the others are not given in the literature as far as the author knows.

\subsection{Close-to-convex functions with argument $\lambda$}

Definition 2. A function $f \in \mathcal{A}_{1}$ is said to be close-to-convex (denoted by $f \in \mathcal{C} \mathcal{L})$ if there exist a starlike function $g$ and a real number $\lambda \in(-\pi / 2, \pi / 2)$ such that

$$
\frac{z f^{\prime}}{g} \in \mathcal{P}_{\lambda}
$$

If we specify the real number $\lambda$ in the above definition, the corresponding function is called a close-to-convex function with argument $\lambda$ and we denote the class of these functions by $\mathcal{C} \mathcal{L}(\lambda)$ (see [5, II, Definition 11.4]). Note that the union of class $\mathcal{C} \mathcal{L}(\lambda)$ over $\lambda \in(-\pi / 2, \pi / 2)$ is precisely $\mathcal{C} \mathcal{L}$. The sharp coefficient bounds of the class $\mathcal{C} \mathcal{L}(\lambda)$ are known (see [27]). 
Lemma 6 (See [3]). Let $f \in \mathcal{S}^{*}$. Then

$$
\frac{r}{(1+r)^{2}} \leq|f(z)| \leq \frac{r}{(1-r)^{2}}
$$

where $r=|z| \in(0,1)$. Equalities occur if and only if $f$ is a suitable rotation of the Koebe function $k(z)=z /(1-z)^{2}$.

By applying Theorem 5 and Lemma 6, we can get a sharp distortion theorem for $\mathcal{C} \mathcal{L}(\lambda)$.

Theorem 8. Let $f(z) \in \mathcal{C} \mathcal{L}(\lambda)$ for a real constant $\lambda \in(-\pi / 2, \pi / 2)$. Then

$$
\frac{1}{A(\lambda, r)(1+r)^{2}} \leq\left|f^{\prime}(z)\right| \leq \frac{A(\lambda, r)}{(1-r)^{2}},
$$

where $A(\lambda, r)$ is given in (5) and $r=|z|<1$. The inequalities are sharp with the extremal functions $f(z)$ satisfying

$$
f^{\prime}(z)=\frac{1+e^{-2 i \lambda} x z}{(1-y z)^{2}(1-x z)}
$$

for $|x|=|y|=1$.

Remark 4. Theorem 8 improves the distortion theorem of close-to-convex functions (see [3]) since the real-valued function $A(\lambda, r)$ is symmetric in $\lambda$ with respect to the origin and

$$
\frac{1-r}{1+r} \leq A(\lambda, r) \leq \frac{1+r}{1-r}
$$

for any $\lambda \in(-\pi / 2, \pi / 2)$.

Though it is easy to deduce the growth theorem of close-to-convex functions with argument $\lambda$ from Theorem 8 , we omit it here since the form is not very esthetics.

\subsection{Analytic functions whose derivative is in $\mathcal{P}_{\boldsymbol{\lambda}}$}

Let $\mathcal{D}(\lambda)=\left\{f \in \mathcal{A}_{1}: f^{\prime} \in \mathcal{P}_{\lambda}\right\}$ for $-\pi / 2<\lambda<\pi / 2$. It is easy to see that $\mathcal{D}(\lambda) \subset \mathcal{C} \mathcal{L}(\lambda)$, thus $\mathcal{D}(\lambda) \subset \mathcal{S}$. Some properties of $\mathcal{D}(\lambda)$ can be deduced from those of $\mathcal{D}(0)$ which have been studied in [7], [17] and so on. We shall only present a distortion theorem which is a direct consequence of Theorem 5 .

Theorem 9. Let $f \in \mathcal{D}(\lambda)$. Then

$$
1 / A(\lambda, r) \leq\left|f^{\prime}(z)\right| \leq A(\lambda, r)
$$

where $r=|z|<1$ and $A(\lambda, r)$ is given by (5). These inequalities are sharp with the extremal function

$$
f(z)=-\left(1+e^{-2 i \lambda}\right) \log (1-z)-e^{-2 i \lambda} z .
$$


For a locally univalent function $f$ on $\mathbb{D}$, the hyperbolic norm of the preSchwarzian derivative $T_{f}=f^{\prime \prime} / f^{\prime}$ is defined by

$$
\left\|T_{f}\right\|=\sup _{|z|<1}\left(1-|z|^{2}\right)\left|T_{f}(z)\right| \text {. }
$$

Since each function in $\mathcal{P}_{\lambda}$ is a Gelfer function, by Gelfer's theorem (see [12, Theorem 2.4]), we have for each function $f \in \mathcal{D}(\lambda)$,

$$
\left\|T_{f}\right\| \leq 2 \text {. }
$$

Our next result shows that this estimate is sharp for the class $\mathcal{D}(\lambda)$, and the extremal functions are also given.

Theorem 10. Let $f \in \mathcal{D}(\lambda)$. Then

$$
\left\|T_{f}\right\| \leq 2 \text {. }
$$

This bound is sharp for each $\lambda \in(-\pi / 2, \pi / 2)$ with the extremal function $f$ given in (13).

Proof. For $f \in \mathcal{D}(\lambda)$, we have $f^{\prime} \in \mathcal{P}_{\lambda}$, thus in view of Theorem 6 ,

$$
\left|\frac{z f^{\prime \prime}(z)}{f^{\prime}(z)}\right| \leq M(\lambda,|z|),
$$

where $M(\lambda, r)$ is given in (6). Remark 3 gives that $M(\lambda, r) \leq M(0, \lambda)=$ $2 r /\left(1-r^{2}\right)$, therefore we have $\left\|T_{f}\right\| \leq 2$. The sharpness can be obtained by observing that $M(\lambda, r)=M(0, \lambda)$ if $r \geq|\tan (\lambda / 2)|$.

Note that the hyperbolic norm of $f \in \mathcal{D}(0)$ was obtained by Nunokawa [18] as well. It is known that (cf. [11]) $f$ is bounded if $\left\|T_{f}\right\|<2$ and the bound depends only on the value of $\left\|T_{f}\right\|$.

Acknowledgements. The author is grateful to Professor Toshiyuki Sugawa for his constant encouragement and useful discussions during the preparation of this paper. Without his help, this paper would never be finished. The author also thanks the referee for several helpful suggestions and comments which improve this paper.

\section{References}

[1] O. P. Ahuja and H. Silverman, A survey on spiral-like and related function classes, Math. Chronicle 20 (1991), 39-66.

[2] S. D. Bernardi, New distortion theorems for functions of positive real part and applications to the partial sums of univalent convex functions, Proc. Amer. Math. Soc. 45 (1974), 113-118.

[3] P. L. Duren, Univalent Functions, Grundlehren Math. Wiss. 259, Springer-Verlag, New York, 1983

[4] S. Gelfer, On the class of regular functions which do not take on any pair of values $\omega$ and $-\omega$, Mat. Sb. 19 (1946), 33-46.

[5] A. W. Goodman, Univalent Functions. Vol. II, Mariner Publishing Co. Inc., 1983.

[6] I. Graham and G. Kohr, Geometric Function Theory in One and Higher Dimensions, Marcel Dekker, Inc., New York, 2003. 
[7] F. Gray and St. Ruscheweyh, Functions whose derivatives take values in a half-plane, Proc. Amer. Math. Soc. 104 (1988), no. 1, 215-218.

[8] D. J. Hallenbeck and T. H. MacGregor, Linear Problems and Convexity Techniques in Geometric Function Theory, Monographs and Studies in Mathematics, 22. Pitman (Advanced Publishing Program), Boston, MA, 1984.

[9] G. Herglotz, Über Potenzreihen mit positivem, reellen Teil in Einheitskreis, Ber. Verh. Sachs. Akad. Wiss. Leipzig (1911), 501-511.

[10] F. Holland, The extreme points of a class of functions with positive real part, Math. Ann. 202 (1973), 85-87.

[11] Y. C. Kim and T. Sugawa, Growth and coefficient estimates for uniformly locally univalent functions on the unit disk, Rocky Mountain J. Math. 32 (2002), no. 1, 179-200.

[12] _ A conformal invariant for nonvanishing analytic functions and its applications, Michigan Math. J. 54 (2006), no. 2, 393-410.

[13] N Norm estimates of the pre-Schwarzian derivatives for certain classes of univalent functions, Proc. Edinb. Math. Soc. (2) 49 (2006), no. 1, 131-143.

[14] _ A note on Bazilevič functions, Taiwanese J. Math. 13 (2009), no. 5, 1489-1495.

[15] R. A. Kortram, The extreme points of a class of functions with positive real part, Bull. Belg. Math. Soc. Simon Stevin 4 (1997), no. 4, 449-459.

[16] R. J. Libera, Some radius of convexity problems, Duke Math. J. 31 (1964), 143-158.

[17] T. H. MacGregor, Functions whose derivative has a positive real part, Trans. Amer. Math. Soc. 104 (1962), 532-537.

[18] M. Nunokawa, On the univalency and multivalency of certain analysis functions, Math. Z. 104 (1968), 394-404.

[19] M. S. Robertson, Variational methods for functions with positive real part, Trans. Amer. Math. Soc. 102 (1962), 82-93.

[20] _ Extremal problems for analytic functions with positive real part and applications, Trans. Amer. Math. Soc. 106 (1963), 236-253.

[21] _ Radii of star-likeness and close-to-covexity, Proc. Amer. Math. Soc. 16 (1965), $847-852$.

[22] St. Ruscheweyh, Convolution in geometric function theory, Sém. Math. Sup. 83, University of Montréal, Montréal, Québec, Canada 1982.

[23] St. Ruscheweyh and V. Singh, On certain extremal problems for functions with positive real part, Proc. Amer. Math. Soc. 61 (1976), no. 2, 329-334.

[24] K. Sakaguchi, A variational method for functions with positive real part, J. Math. Soc. Japan 16 (1964), 287-297.

[25] I. Schur, Über Potenzreihen, die im Innern des Einheitskreises beschränkt sind, J. Reine Angew. Math. 147 (1917), 205-232; 148 (1918), 122-145

[26] L. Špaček, Contribution à la théorie des fonctions univalentes, Casopis Pěst. Mat.-Fys. 62 (1932), 12-19.

[27] L.-M. Wang, Coefficient estimates for close-to-convex functions with argument $\lambda$, Bull. Berg. Math. Soc. (to appear).

[28] S. Yamashita, Gel'fer functions, integral means, bounded mean oscillation, and univalency, Trans. Amer. Math. Soc. 321 (1990), no. 1, 245-259.

[29] V. A. Zmorovič, On bounds of convexity for starlike function of order $\alpha$ in the circle $|z|<1$ and in the circular region $0<|z|<1$, Mat. Sb. 68 (110) (1965), 518-526; English transl., Amer. Math. Soc. Transl. (2) 80 (1969), 203-213.

[30] - On the bounds of starlikeness and univalence in certain classes of functions regular in the circle $|z|<1$, Ukrain. Mat. Ž. 18 (1966), 28-39; English transl., Amer. Math. Soc. Transl. (2) 80 (1969), 227-242. 
Division OF MATHEMATICS

Graduate School of Information Sciences

TOHOKU UNIVERSITY

6-3-09 Aramaki-Aza-Aoba, Aoba-Ku, Sendai, Miyagi 980-8579, Japan

E-mail address: rime@ims.is.tohoku.ac.jp 